\title{
Two British women studies replicated the association between the Val66Met polymorphism in the brain-derived neurotrophic factor (BDNF) and BMI
}

Yin Yao Shugart*,1,2, Lina Chen ${ }^{2}$, Ian NM Day ${ }^{3}$, Sarah J Lewis ${ }^{2}$, Nicholas J Timpson ${ }^{3}$, Wei Yuan ${ }^{4}$, Mohammad Reza Abdollahi $^{2}$, Sue M Ring ${ }^{5}$, Shah Ebrahim ${ }^{6}$, Jean Golding ${ }^{5}$, Debbie A Lawlor ${ }^{3}$ and George Davey-Smith ${ }^{3}$

${ }^{1}$ Department of Epidemiology, Johns Hopkins University, Baltimore, MD, USA; ${ }^{2}$ Department of Social Medicine, University of Bristol, Bristol, UK; ${ }^{3} \mathrm{MRC}$ Centre for Causal Analyses in Translational Epidemiology, University of Bristol, Bristol, UK; ${ }^{4}$ Department of Clinical Science at South Bristol (Obstetrics \& Gynaecology), University of Bristol, Bristol, UK; ${ }^{5}$ Department of Social Medicine/ALSPAC, University of Bristol, Bristol, UK; ${ }^{6}$ Department of Public Health, London School of Hygiene \& Tropical Medicine, London, UK

The goal of this study is to investigate the relationship between the Val66Met polymorphism in the brainderived neurotrophic factor (BDNF) and body mass index (BMI) in two sizable and well-characterized populations of British women: the British Women's Heart and Health Study (BWHHS) (age 60-79 years) and the mothers from the Avon Longitudinal Study of Parents and Children (age 16-44 years). We genotyped the Val66Met polymorphism (rs6265) in these two populations, and conducted a linear regression analysis to test for an association between this polymorphism and BMI. Both study populations indicated an association between BMI and the Val66Met polymorphism, with individuals carrying the Met-Met genotype having a lower mean BMI than those with the Val-Met or Val-Val genotypes (in the BWHHS): mean BMI difference $=-0.911 \mathrm{~kg} / \mathrm{m}^{2}, 95 \%$ confidence interval $(\mathrm{CI}):-1.70$ to $-0.12, P=0.023 ;$ in the mothers from the Avon Longitudinal Study of Parents and Children (ALSPAC): mean BMI difference $=-0.57 \mathrm{~kg} / \mathrm{m}^{2}, 95 \% \mathrm{Cl}:-1.08$ to $\left.-0.054, P=0.03\right)$. In a pooled analysis of these two studies, together with one further published study that provided data in a suitable format for inclusion in our meta-analysis, we found a pooled difference of $-0.76(95 \% \mathrm{Cl}:-1.16,-0.036)$ for adult women; $I^{2}$-test for heterogeneity $=51 \%, P=0.13$. Our study indicated an association between $B D N F$ and $B M I$ in two general population studies of women. The exact role of $B D N F$ in weight regulation merits further investigation. European Journal of Human Genetics (2009) 17, 1050-1055; doi:10.1038/ejhg.2008.272; published online 11 February 2009

Keywords: BWHHS; ALSPAC

*Correspondence: Dr YY Shugart, Department of Epidemiology, Johns Hopkins Bloomberg School of Public Health, Johns Hopkins University, 615 N. Wolfe Street, Room E6516, Baltimore, MD 21205, USA. Tel: + 1301443 2037; Fax: + 1301402 4740;

E-mails: kay1yao@mail.nih.gov and yin.yao@bristol.ac.uk

Received 23 October 2008; revised 26 September 2008; accepted 17 December 2008; published online 11 February 2009

\section{Introduction}

It has been documented that the brain-derived neurotrophic factor $(B D N F)$ plays an essential role in the development, survival and differentiation of neurons through its receptor, tropomyosin-related kinase B $(\operatorname{TrkB}){ }^{1}$ $B D N F$ has also been implicated in a range of behaviors, including hyperphagia and hyperactivity, and is associated with obesity in several types of knockout mice models. ${ }^{2-4}$ Taken together, these studies implicate 
the role of $B D N F$ in weight regulation (related to eating disorders (EDs) and obesity) and in the development of behavioral disorders (related to anxiety, hyperactivity and aggressive behaviors).

$B D N F$ 's involvement in the development of obesity in humans has been highlighted by two earlier studies. ${ }^{5,6}$ Furthermore, in humans, a BDNF contribution to the etiology of EDs has been documented by using a casecontrol study design in six European populations. ${ }^{7}$ Specifically, they reported a strong association between the Met66 variant within the BDNF gene and all ED sub-phenotypes, including anorexia (AN), restricting AN (ANR), binge-eating/purging $\mathrm{AN}$ and bulimia nervosa (BN). They also observed an association between another BDNF variant ' $-270 \mathrm{C}>\mathrm{T}$ ' and $\mathrm{BN}$ and late age-at-onset of weight loss. ${ }^{7}$ In a later study, to replicate their earlier findings, Ribases et al genotyped both genetic variants in an additional 453 ED trios recruited from six European centers and reported an over-transmission of the $-270 \mathrm{C} / \mathrm{Met} 66$ haplotype to the offspring affected with ANR. ${ }^{8}$ In comparison, the relationship between genetic variants in $B D N F$ and obesity in humans has been less well studied. Gunstad et.al. ${ }^{9}$ first investigated a direct effect of the Val66Met polymorphism (rs6265) on mean BMI in humans.They tested an association between the BDNF Val66Met polymorphism and BMI in a healthy population of 481 adults aged between 18-82 years. ${ }^{9}$ After adjusting for gender, their analysis suggested that individuals with the Met-Met genotype had a lower mean BMI than those with the Val-Met or Val-Val genotypes. This association was statistically significant for women, but in the analyses combining women and men, and adjusting for gender, it did not reach conventional levels of statistical significance. ${ }^{9}$ Taken together, these findings could be interpreted as suggesting that the Met-Met genotype may modify energy balance pattern and, therefore, these individuals are less likely to develop obesity or EDs. This effect may be particularly so for women.

Our aim in this study is to further investigate the relationship between the genetic variants in BDNF and BMI by genotyping the same genetic variants in $B D N F$ in two sizable and well-characterized populations of British women: (1) the British Women's Heart and Health Study (BWHHS); and (2) the mothers from the Avon Longitudinal Study of Parents and Children (ALSPAC). ${ }^{10,11}$ Both studies have considerably greater statistical power than the original published study of this association, ${ }^{9}$ and than a second published study that also reported a null association. $^{12}$

\section{Participants and methods}

\section{The British Women's Heart and Health Study}

The BWHHS is a prospective cohort study established primarily to determine the causes and consequences of heart disease in women. Between 1999 and 2001, 4286 women in the age range of 60-79 were randomly selected from 23 British towns to take part in this study. Of the 4286 participants, eight refused consent for genetic testing, 349 did not donate blood samples and 29 were described by the examining nurses as being non-white. Of the remaining 3900 women, 3631 individuals were successfully genotyped and also had information on BMI; these women were included in this analysis. All the participants in this study were interviewed, examined, completed medical questionnaires and had detailed reviews of their medical records. The full details of the selection of study participants and their measurements have been reported earlier ${ }^{13}$

The body mass index of all participants was calculated from weight (assessed in light clothing) and standing height, which were measured in the clinic by research nurses using standard procedures. Participants were also asked to report their smoking status and were classified into three groups: non-smoker, past-smoker and current smoker. Daily alcohol consumption (consuming alcohol on most days or everyday were classified as high alcohol consumption) and physical activity (subjects with $<2 \mathrm{~h}$ of moderate or vigorous activity per week were defined as physically inactive) were also obtained from the interview or questionnaires. The occupational social class of the head of the household was derived from the participant's report of both her and her spouse's occupation, using the UK Registrar General's Classification (social class I indicates professional; II indicates managerial/technical; III indicates skilled non-manual; IIIM indicates skilled manual; IV indicates partly skilled manual; and $\mathrm{V}$ indicates unskilled manual worker).

Local medical research ethics committee approvals were obtained for the BWHHS. Participants were asked for informed consent to carry out anonymized genetic tests on stored blood. The eight women who declined to give consent were not included in this study.

\section{Mothers from the Avon Longitudinal Study of Parents and Children}

The ALSPAC study is designed to observe the ways in which different aspects of the environment affect the development and health of the child and parents, and the extent to which such influences are affected by genetic susceptibility. For the purpose of this study, we only used the blood samples from the mothers from ALSPAC. It is a geographically based cohort that recruited pregnant women residing in Avon with an expected delivery date between 1 April 1991 and 31 December 1992. A total of 14541 pregnant women were initially enrolled, which represents $80-90 \%$ of the eligible population ${ }^{10}$; see www.alspac.bris.ac.uk for further information about the ALSPAC cohort. Ethical approval was obtained from the ALSPAC Law and Ethics Committee and from local research ethics committees. 
Body Mass Index was calculated using self-reported weight and height obtained from a questionnaire completed in early pregnancy, in which the mothers were asked to report their weight and height immediately before their pregnancy. The occupational social class was obtained using the same method described for BWHHS.

Of a total of 15311 mothers who participated in the ALSPAC study, 12794 described themselves as European British. Of these women, 7779 donated a blood sample and 6478 had data on both genotype and BMI, and were included in our analysis.

\section{DNA preparation and genotyping}

DNA from peripheral blood was extracted and processed as described earlier. $^{11}$

All genotyping was carried out by KBioscience (http:// www.kbioscience.co.uk). Single-nucleotide polymorphisms (SNPs) were genotyped using the KASPar chemistry, which is a competitive allele-specific PCR-SNP genotyping system using FRET quencher cassette oligos (http:// www.kbioscience.co.uk/genotyping/genotyping_chemistry. html). Blind duplicates, plate-identifying blank wells and the Hardy-Weinberg equilibrium (HWE) tests were used as quality control tests. Further, the same assay and conditions were used to genotype our samples.

\section{Statistical analysis}

The Hardy-Weinburg Equilibrium was assessed using Pearson's $\chi^{2}$-test. The distribution of non-genetic variables, such as age, socioeconomic position and consumption of tobacco and alcohol, by genotype is presented. These analyses were undertaken to determine whether any of these characteristics might confound the association of genotype with BMI. The $\chi^{2}$-test was used for categorical data and Student's $t$-test was used for continuous data to determine statistical evidence for variations in the distributions of these variables by genotype. Consistent with earlier studies, we a priori tested a recessive genetic model for the association of BDNF with BMI, that is, we assessed the mean difference in BMI between women with the Met-Met genotype and those with either Val-Met or Val-Val. We used student's $t$-test to examine unadjusted associations between these two groups defined by genotype and BMI. To adjust for covariables, we performed a multivariable linear regression model. We a priori decided that we would adjust for age and, in addition, any other covariables that were found in either study to be associated with genotype (and BMI). As noted in Table 1 social class in the BWHHS study and alcohol consumption in the ALSPAC study were found to be associated with genotype and were, therefore, included in the linear regression models for their respective studies, along with age (included in both studies). The resultant regression coefficient for genotype gives the mean difference in BMI between the two groups, having controlled for other covariables. We tested the underlying assumption of a linear regression model - that the outcome (in this case, BMI) is a linear function of the regression parameters of the model - by examining the distribution of residuals from the model. (Normally distributed residuals indicate that the linearity assumption of the model is not violated.) ${ }^{14}$ A normal plot showed that the residuals were normally distributed

Table 1 Baseline characteristics of participants from BWHHS and ALSPAC by BDNF genotypes (mean \pm s.d. for continuous data and total number for categorical data)

\begin{tabular}{|c|c|c|c|c|c|c|}
\hline \multirow[b]{2}{*}{ Val66Met } & \multicolumn{3}{|c|}{ BWHHS } & \multicolumn{3}{|c|}{ ALSPAC } \\
\hline & $V V / V M$ & $M M$ & P-value & $V V / V M$ & $M M$ & P-value \\
\hline $\begin{array}{l}\text { Number } \\
\text { Age (years) }\end{array}$ & $\begin{array}{c}3468 \\
68.84 \pm 5.5\end{array}$ & $\begin{array}{c}163 \\
69.25 \pm 5.6\end{array}$ & 0.350 & $\begin{array}{c}6253 \\
28.47 \pm 4.7\end{array}$ & $\begin{array}{c}225 \\
28.48 \pm 4.6\end{array}$ & 0.996 \\
\hline $\begin{array}{l}\text { Social class } \\
\text { Manual } \\
\text { Non-manual }\end{array}$ & $\begin{array}{l}1965 \\
1503\end{array}$ & $\begin{array}{l}80 \\
83\end{array}$ & 0.057 & $\begin{array}{l}2754 \\
2996\end{array}$ & $\begin{array}{r}103 \\
96\end{array}$ & 0.284 \\
\hline $\begin{array}{l}\text { Smoking status } \\
\text { Non-smoker } \\
\text { Smoker }\end{array}$ & $\begin{array}{r}1937 \\
1,529\end{array}$ & $\begin{array}{r}102 \\
61\end{array}$ & 0.092 & $\begin{array}{l}3171 \\
2948\end{array}$ & $\begin{array}{l}106 \\
112\end{array}$ & 0.353 \\
\hline $\begin{array}{l}\text { Daily drinking } \\
\text { No } \\
\text { Yes }\end{array}$ & $\begin{array}{r}2850 \\
615\end{array}$ & $\begin{array}{r}128 \\
35\end{array}$ & 0.226 & $\begin{array}{r}5407 \\
720\end{array}$ & $\begin{array}{r}202 \\
16\end{array}$ & 0.046 \\
\hline $\begin{array}{l}\text { Physical activity } \\
\text { Active } \\
\text { Inactive }\end{array}$ & $\begin{array}{r}2800 \\
659\end{array}$ & $\begin{array}{r}132 \\
31\end{array}$ & 0.992 & $\begin{array}{l}2353 \\
2823\end{array}$ & $\begin{array}{r}77 \\
113\end{array}$ & 0.180 \\
\hline
\end{tabular}

The Va166Met genotypes has been labelled as MM: Met-Met; VM: Val-Met; VV: Val-Val.

${ }^{a}$ Cut-off of physical activity is $2 \mathrm{~h}$ physical exercise per week in BWHHS and one physical exercise per week in ALSPAC. 
and, therefore, the linearity assumption was not violated in this model.

\section{Meta-analysis}

To increase precision and also to explore whether any association might differ between different study populations, we decided to conduct a meta-analysis of our two primary study results and other relevant published results. We searched the literature for earlier publications of this association using the following terms: BDNF, Brain-derived neurotrophic factor, in combination with BMI, body mass index. We also searched the results of earlier published genome-wide association studies of BMI or obesity.

As the sampling frame and population characteristics (particularly with respect to age) of the two primary study populations (BWHHS and ALSPAC) are different, and as we also wished to include results from other published studies if they provide individual participant data, we decided $a$ priori to pool effect estimates rather than individual participant data. We tested between-study heterogeneity by means of Cochran's $Q$-test and quantified any heterogeneity using the $I^{2}$ measure. ${ }^{15}$ (As pointed by Higgins et al (2003), a better approach to quantify the heterogeneity effect can be used to measure the degree of inconsistency in results from various investigations.) ${ }^{15}$ An $I^{2}$ value of zero indicated no heterogeneity and larger values indicated evidence of heterogeneity. The results showed borderline heterogeneity $\left(I^{2}=51 \%\right)$. Hence we conducted both a random and a fixed-effect meta-analysis; the results for these two were essentially the same and the fixed effect meta-analysis is presented. All analyses were conducted using STATA version 9.2 .

\section{Results}

Of the characteristics tested, only alcohol consumption in the ALSPAC mothers was associated with genotype (Table 1). There was also weak statistical evidence of an association between social class and genotype in the BWHHS study. Therefore, both these variables were included in the final genetic analysis model.
In BWHHS, approximately $64.5 \%$ of the total study population $(n=2346)$ were Val-Val homozygotes, $30.9 \%$ $(n=1122)$ were Val-Met heterozygotes and $4.49 \%$ $(n=163)$ were Met-Met homozygotes. There was no deviation from HWE, $P=0.053$. In ALSPAC, approximately $65.8 \%$ of the total study population $(n=4261)$ were Val-Val homozygotes, 30.8\% $(n=1992)$ were Val-Met heterozygotes and 3.47\% $(n=225)$ were Met-Met homozygotes. There was no deviation from HWE, $P=0.68$.

The associations of genotype with BMI for both studies are presented in Table 2 . As our primary outcome variable is a quantitative trait, and we conducted a liner regression analysis, we reported the 'mean difference' in BMI of two different groups (carriers of Met-Met $v s$ carriers of Val-Val and Val-Met).

Both study populations indicated an association between BMI and the Val66Met polymorphism, with individuals carrying the Met-Met genotype having a lower mean BMI than those with the Val-Met or Val-Val genotypes. Associations did not change substantially with adjustment for alcohol consumption (ALSPAC) or social class (BWHHS), or both, both of which had shown weak associations with genotype (see Table 1) or for adjustment with age in both studies (adjusted $P$-values presented in Table 2 ).

We were able to obtain data in a relevant format for inclusion in our meta-analyses only from one of these studies. ${ }^{9}$ Figure 1 shows the meta-analysis combining data

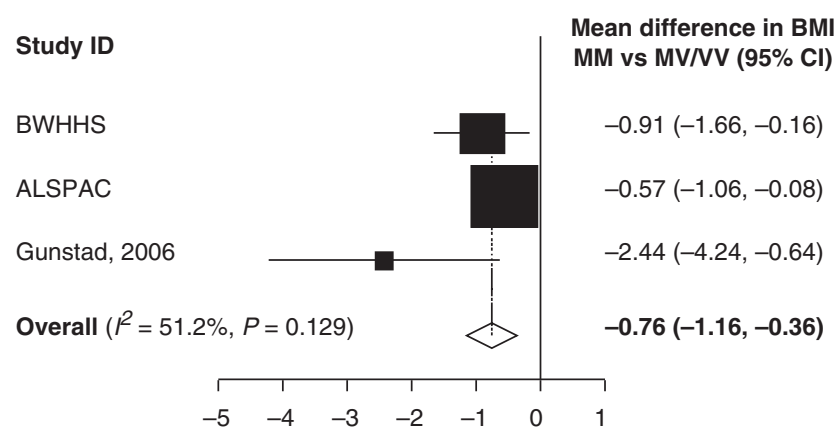

Figure 1 The mean difference in BMI by comparing Met-Met to Met-Val/Val-Val genotype in two cohort studies, BWHHS and ALSPAC.

Table 2 BMl comparison by genotype and study group

\begin{tabular}{lccccc}
\hline & VM/VV & $M M$ & Mean difference in BMI MM vs VM/MM (95\%Cl) & P-value & Adjusted P \\
\hline BWHHS & 3468 & 163 & & $0.911(-1.70,-0.12)$ & 0.023 \\
BMI (mean \pm s.d.) & $27.63 \pm 5.02$ & $26.72 \pm 4.78$ & & \\
ALSPAC & 6253 & 225 & $-0.57(-1.08,-0.054)$ & 0.030 \\
BMI (mean \pm s.d.) & $23.04 \pm 3.87$ & $22.47 \pm 3.65$ & 0.021 \\
\hline
\end{tabular}

Abbreviations: ALSPAC, avon longitudinal study of parents and children; BMI, body mass index; BWHHS, British women's heart and health study. The Va166Met genotypes has been labelled as MM: Met-Met; VM: Val-Met; VV: Val-Val.

Mean difference in BMI is the difference between two normalized means of two comparison groups (carriers of MM vs carriers of MM and MV) and are given in $\mathrm{Kg} / \mathrm{m}^{2}$. The mean values divided by an estimate of the within-group standard deviation have shown. Ages of participants were used as covariates in the association model in both studies, while alcohol consumption in ALSPAC and social class in BWHHS, both of which had shown weak associations with genotype (see Table 1), were also included as covariates in the adjusted $P$-values. 
from ALSPAC, BWHHS and the data for women reported by Gunstad et al. ${ }^{9}$ The pooled estimate indicated a mean difference of $0.76 \mathrm{~kg} / \mathrm{m}^{2}$ (95\% confidence interval (CI): 0.36 , 1.16), $P<0.001$, comparing women with the Met-Met genotype with all other women (Figure 1).

\section{Discussion}

Our study strongly indicated an association between the Met-Met genotype in BDNF and lower BMI in two general population studies of European women, both of which have considerably greater sample sizes than the initial study carried out by Gunstad et al (2006). Although the gender-combined association finding by Gunstad et al did not reach the statistical significance level required to claim an association finding, the effect they reported was in the same direction as we reported here. Furthermore, their findings for women only appear consistent with our findings in ALSPAC and BWHHS women.

In general, population-based association studies are more powerful than family-based linkage studies for detecting genes with modest effects and lower penetrance underlying complex human traits. ${ }^{16}$ However, the possibility of generating false positive findings from epidemiological studies is acknowledged. ${ }^{17}$ Therefore, replication studies are mandatory to verify genetic association findings for complex traits. This study generates exciting findings for an association between BMI and a genetic variant in $B D N F$, which further establishes the role of Val66Met in variations in BMI and possibly in the development of obesity in women. We would like to note that both studies are of British origin and the genotyping was conducted by the same commercial company using the same PCR conditions. The similar magnitude of effect, despite the populations having different age and BMI distributions, increases the likelihood that these are not chance findings or explained by bias. Our findings are also broadly consistent with those for women in the study by Gunstad et al, though we acknowledge that those results suggest an even stronger association, and the small sample size of that study, with consequent wide CI, limits the power of our test for heterogeneity between it and our findings in ALSPAC and BWHHS. (As can be seen in Figure 1, the study by Gunstad et al accounts for nearly 51\% variation between studies.) In the ALSPAC mothers, weight and height were based on self-report and a number of studies have reported systematic reporting bias in a variety of populations for these characteristics, such that those who are shorter tend to overestimate their height and those who are fatter tend to underestimate their weight. ${ }^{18,19}$ Individuals will not be aware of their BDNF status and, therefore, it is unlikely that this misreporting will be differential by genotype and will lead to important bias. Furthermore, the similarity in effect between our two studies (with direct measurement of BMI in BWHHS) suggests that the use of self-reported data in ALSPAC is unlikely to have importantly biased our reported findings from this study.

Despite our results in two large studies, it is important to note that other studies have failed to find an association between BDNF and BMI. The study by Friedel et al ${ }^{12}$ had a small sample size and may have had insufficient statistical power to exclude an effect. Two published genome-wide association studies (GWAS) failed to detect Val66Met as associated with BMI. ${ }^{20,21}$ One possible explanation for this observation is that studies with different design and/or conducted in different populations may lead to the discovery of different genetic variants that are associated with obesity-related traits. GWAS, because of their nature, require more stringent criteria for statistically testing the candidate gene approaches that are, in theory, more hypothesis driven. In the large number of associations tested, only results that show significant GWAS associations are presented more fully and we were unable to obtain data from these studies in a format that would enable us to include them in our meta-analysis. Thus, although our results suggest an association in women, we do not have sufficient information to formally test whether there is any true difference in the association between women and men, and it is possible that our findings in women are because of chance. Further large studies that include large numbers of both men and women are required to examine this. Studies exploring the mechanisms by which BDNF might influence BMI are also warranted.

Finally, we would like to acknowledge another limitation of this study. Owing to limited funding, we were unable to examine possible population stratification using additional unrelated SNPs. It is possible that there may exist a subtle level of population stratification because there seems to be a difference in the distribution of BDNF genotypes between two populations $(P<0.05)$. In the future, we hope to have genome-wide data in the ALSPAC population that will enable us to explore this possibility further.

In conclusion, our findings provide some further evidence that $B D N F$ is associated with variation in BMI in women. Further large studies are required to determine whether there is a true gender difference in this association and possible mechanism.

\footnotetext{
Acknowledgements

The BWHHS is funded by the UK Department of Health and the British Heart Foundation.

Funding for the ALSPAC study has come from a wide variety of sources, including the University of Bristol, Welcome Trust, the Medical Research Council, UK Government Departments such as MAFF, Department of Health, Department for Education and the Health and Safety Executive. Other sources of funds include Medical Research Charities such as the Welcome Trust, the National Asthma Campaign, and American sources such as the National Institutes of
} 
Health and the March of Dimes charity.Debbie A Lawlor is funded by a UK Career Scientist Award. Nicholas I Timpson is funded by a UK Medical Research Council (MRC) studentship.The views expressed in this study are those of the authors and not necessarily of any funding agency. No funding agency has influenced the data analysis or its interpretation. This work was written while Yin Yao Shugart was working at Johns Hopkins University. She is currently working at the Genomic Research branch at NIMH. The views expressed in this article do not necessarily represent the views of the NIMH, NIH, HHS, or the United States Government.

We would also like to thank the participants in both studies and the large number of staff who have collected, cleaned and managed data for both studies.

\section{References}

1 Lebrun B, Bariohay B, Moyse E, Jean A: Brain-derived neurotrophic factor (BDNF) and food intake regulation: a minireview. Auton Neurosci 2006; 126-127: 30-38.

2 Lyons WE, Mamounas LA, Ricaurte GA et al: Brain-derived neurotrophic factor-deficient mice develop aggressiveness and hyperphagia in conjunction with brain serotonergic abnormalities. Proc Natl Acad Sci USA 1999; 96: 15239-15244.

3 Kernie SG, Liebl DJ, Parada LF: BDNF regulates eating behavior and locomotor activity in mice. EMBO J 2000; 19: 1290-1300.

4 Rios M, Fan G, Fekete C et al: Conditional deletion of brainderived neurotrophic factor in the postnatal brain leads to obesity and hyperactivity. Mol Endocrinol 2001; 15: 1748-1757.

5 El-Gharbawy AH, Adler-Wailes DC, Mirch MC et al: Serum brain-derived neurotrophic factor concentrations in lean and overweight children and adolescents. J Clin Endocrinol Metab 2006; 91: 3548-3552.

6 Gray J, Yeo GS, Cox JJ et al: Hyperphagia, severe obesity, impaired cognitive function, and hyperactivity associated with functional loss of one copy of the brain-derived neurotrophic factor (BDNF) gene. Diabetes 2006; 55: 3366-3371.

7 Ribases M, Gratacos M, Fernandez-Aranda F et al: Association of BDNF with anorexia, bulimia and age of onset of weight loss in six European populations. Hum Mol Genet 2004; 13: 1205-1212.

8 Ribases M, Gratacos M, Fernandez-Aranda F et al: Association of BDNF with restricting anorexia nervosa and minimum body mass index: a family-based association study of eight European populations. Eur J Hum Genet 2005; 13: 428-434.

9 Gunstad J, Schofield P, Paul RH et al: BDNF Val66Met polymorphism is associated with body mass index in healthy adults. Neuropsychobiology 2006; 53: 153-156.

10 Golding J, Pembrey M, Jones R: ALSPAC-the Avon Longitudinal Study of Parents and Children.I. Study Methodology. Paediatr Perinat Epidemiol 2001; 15: 74-87.

11 Jones RW, Ring S, Tyfield L et al: A new human genetic resource: a DNA bank established as part of the Avon Longitudinal Study of Pregnancy and Childhood (ALSPAC). Eur J Hum Genet 2000; 8: 653-660.

12 Friedel S, Horro FF, Wermter AK et al: Mutation screen of the brain derived neurotrophic factor gene (BDNF): identification of several genetic variants and association studies in patients with obesity, eating disorders, and attention-deficit/hyperactivity disorder. $A m$ J Med Genet B Neuropsychiatr Genet 2005; 132B: 96-99.

13 Lawlor DA, Bedford C, Taylor M, Ebrahim S: Geographical variation in cardiovascular disease, risk factors, and their control in older women: British Women's Heart and Health Study. J Epidemiol Community Health 2003; 57: 134-140.

14 Draper NR, Smith H: Applied Regression Analysis. New York: Wiley, 1998.

15 Higgins JP, Thompson SG, Deeks JJ, Altman DG: Measuring inconsistency in meta-analyses. BMJ 2003; 327: 557-560.

16 Risch N, Merikangas K: The future of genetic studies of complex human diseases. Science 1996; 273: 1516-1517.

17 Colhoun HM, McKeigue PM, Davey Smith G: Problems of reporting genetic associations with complex outcomes. Lancet 2003; 361: $865-872$.

18 Rowland ML: Self-reported weight and height. Am J Clin Nutr 1990; 52: $1125-1133$.

19 Lawlor DA, Bedford C, Taylor M, Ebrahim S: Agreement between measured and self-reported weight in older women. Results from the British Women's Heart and Health Study. Age Ageing 2002; 31: 169-174.

20 Scuteri A, Sanna S, Chen WM et al: Genome-wide association scan shows genetic variants in the FTO gene are associated with obesity-related traits. PLoS Genet 2007; 3: e115.

21 Fox CS, Heard-Costa N, Cupples LA, Dupuis J, Vasan RS, Atwood LD: Genome-wide association to body mass index and waist circumference: the Framingham Heart Study $100 \mathrm{~K}$ project. $B M C$ Med Genet 2007; 8 (Suppl 1): S18. 